\title{
Hybrid Metaheuristics Web Service Composition Model for QoS Aware Services
}

\author{
P. Rajeswari* and K. Jayashree
}

\author{
Department of Computer Science and Engineering, Rajalakshmi Engineering College, Chennai, 602105, India \\ *Corresponding Author: P. Rajeswari. Email: rajkavin2008@gmail.com \\ Received: 20 May 2021; Accepted: 21 June 2021
}

\begin{abstract}
Recent advancements in cloud computing (CC) technologies signified that several distinct web services are presently developed and exist at the cloud data centre. Currently, web service composition gains maximum attention among researchers due to its significance in real-time applications. Quality of Service (QoS) aware service composition concerned regarding the election of candidate services with the maximization of the whole QoS. But these models have failed to handle the uncertainties of QoS. The resulting QoS of composite service identified by the clients become unstable and subject to risks of failing composition by end-users. On the other hand, trip planning is an essential technique in supporting digital map services. It aims to determine a set of location based services (LBS) which cover all client intended activities quantified in the query. But the available web service composition solutions do not consider the complicated spatio-temporal features. For resolving this issue, this study develops a new hybridization of the firefly optimization algorithm with fuzzy logic based web service composition model (F3L-WSCM) in a cloud environment for location awareness. The presented F3L-WSCM model involves a discovery module which enables the client to provide a query related to trip planning such as flight booking, hotels, car rentals, etc. At the next stage, the firefly algorithm is applied to generate composition plans to minimize the number of composition plans. Followed by, the fuzzy subtractive clustering (FSC) will select the best composition plan from the available composite plans. Besides, the presented F3L-WSCM model involves four input QoS parameters namely service cost, service availability, service response time, and user rating. An extensive experimental analysis takes place on CloudSim tool and exhibit the superior performance of the presented F3L-WSCM model in terms of accuracy, execution time, and efficiency.
\end{abstract}

Keywords: Web service composition; trip planning; hybrid models; firefly algorithm; QoS aware services; fuzzy logic

\section{Introduction}

With the developments of Cloud Computing (CC) and Software as a Service (SaaS), an increased applications and processing resources have been summarized as Web Services (WSs) and provided on the web [1]. The WS consists of cross platform software process, loosely coupled, and flexible, which could

This work is licensed under a Creative Commons Attribution 4.0 International License, which permits unrestricted use, distribution, and reproduction in any medium, provided the original work is properly cited. 
tackle various needs for establishing versatile, agile, and cross enterprise applications [2]. The service composition acts as a solution for the unified combination of business applications to generate novel value-added services over present ones. As several WSs are established online with similar operations and distinct quality features, it turns to be critical in selecting candidate service to attain subsequent composite service (CS) with optimum efficiency. The facilitation has been made and utilized as different applications over the web, Quality of Service (QoS) aware service composition is in great demand, while the WS is understood and defined based on QoS and functional abilities.

In recent years, the WS composition has gained a lot of interest in the research fields. There are 3 stages for QoS aware service composition. Initial design time stage, sub-processes are needed by the CS, and their control, data flow, and interactions are recognized. A recognizable language, WS-BPEL, is generally utilized for modeling abstract compositions. Next, at the pre-processing stage, concrete WS are established to match the abstract services based on function using semantic/syntactic technique [3]. Consequently, a list of functional equivalent WS (i.e., candidate) with distinct QoS is attained for all tasks. During the final run time stage, the decision making WS is depending upon their determined QoS. The whole QoS is defined by the QoS of structural element services which should be optimized to fulfill the user end-to-end QoS limitations. The relevant Combinatorial Problem (CP) is recognized as NP-hard [4].

Though the challenge of QoS-aware WS composition is often tackled by the present investigations [5], a major problem, that is the uncertainty of QoS, is yet unsolved and unconsidered. In real time application areas, WS is regularly subjected to fluctuation and variation because of several unexpected features like congestion and network connectivity. The subsequent QoS of composite WS monitored by clients is not stable and subjected to the risks of failing QoS needs by end-user. On the other hand, Personal trip planning has been established as commonly utilized urban computing service and assisted by digital map service suppliers like Microsoft MapPoint and Google Map. In previous years, trip planning is hot research field, which aims to search for a capable trip for the client using querying search methods, indexing, and effective data modeling with several beneficial methods.

Several methods were presented user defined trip planning queries (TPQ) in an effective way. While computing TPQs, it is essential in considering the activity's limitations. For instance, when the client planning for lunchtime, the suggested trip is estimated to guide the client to a restaurant $[6,7]$. These kinds of activity-based trip planning queries are extensively utilized in spatial crowd sourcing, personal trip suggestion, etc. For TPQ, extensive works were taken for investigating the decision making of a qualified location-based service. But in some instances, a client might have several intentional activities, and probably, they are incapable of supporting access to any individual location-based services closer to the query locations [8]. With this motivation, this study focuses on the design of an effective WS composition model for location awareness.

This study introduces a novel hybridization of the firefly optimization algorithm with fuzzy logic-based web service composition model (F3L-WSCM) in a CC environment for location identification. The presented F3L-WSCM model comprises discovery module which enables the client to provide a query related to trip planning such as flight booking, hotels, car rentals, etc. The presented model involves the firefly (FF) algorithm, which is inspired by the flashing patterns of fireflies for WS composition plan generation. Besides, fuzzy subtractive clustering (FSC) technique is employed for the selection of optimal composition plans from the existing composite plans. A series of simulations were performed on benchmark dataset to demonstrate the promising results of the F3L-WSCM model over the existing methods.

The remaining sections of the paper are planned as follows. Section 2 reviews the recent WS composition planning techniques. Followed by, Section 3 describes the F3L-WSCM model and section 4 validates the experimental results. Finally, Section 5 concludes the study. 


\section{Related Works}

Zhu et al. [9] established the technique that integrates FL with Graph Plan technique. The Fuzzy rules are utilized for evaluating and ranking the services based on user preferences, later, the result with optimum QoS value is chosen and utilized in Graph Plan construction. Alhadithy et al. [10] presented to compose WSs by fuzzy rule in which the electing of WS from Cloud depends upon the QoS which can satisfy client need and limitations. Furthermore, the developed method is offered with a component to monitor the implementation of composing services in the event that any of the composing services becomes inaccessible, the agent would interchange the inaccessible service with new service which matches the client's need and the produced fuzzy rules make novel composition strategy.

Rhimi et al. [11] recommend a solution by exhibiting clients' uncertainty preferences with fuzzy sets. Lastly, they insert conventional features for ensuring global optimization with an effective composition process. Ma et al. [12] proposed a new semantic WS composition technique with fuzzy colored Petri net (FCPN). The FCPN method and algebra definition of service composition basic structure is provided. Sangaiah et al. [13] presented an effective approach to resolve the challenge of WS composition utilizing biogeography-based optimization (BBO). It is a simpler technique with some adjustable parameters. The developed technique provides significant results to this problem.

Da Silva et al. [14] introduced the technique that integrates two concepts of creating novel compositions depending upon data kept in a graph database and later enhancing their quality by utilizing genetic programming. Researches have been accompanied relating the efficiency of recently developed method towards the present work. Outcomes demonstrate that the novel method performs quicker compared to formerly projected work, although it doesn't often attain the similar result quality as the compositions generated by themselves. Xu et al. [15] investigate the challenges of process aware location-based service composition that aims to return a rational trip formed by a group of location-based services that are rationally dispersed in spatial, however, it guarantees each intended activity and their temporal workflow limitations. Mainly, it proposes a set of spatial keywords search based techniques to accelerate the query process.

Wang et al. [16] proposed a novel service composition system depending upon Deep Reinforcement Learning (DRL) for adaptive and large-scale service composition. The projected method is preferable for partial observable service platforms, which makes it better work for real time scenarios. The recurrent neural network (RNN) is accepted to develop RL method that could predict decision criterion and improve the capability to generalize and express. In Mallayya et al. [17], a user preference based WS ranking (UPWSR) technique is developed for ranking the WSs depending upon user preference and QoS factors of the WS. If the user request could not be satisfied by individual atomic service, various present services must be delivered and composed as composition. The presented architecture enables the client for specifying the global and local limitations to complex WSs that enhance the flexibility. The UPWSR method recognizes optimum fit services for every task in the client request and, by selecting the amount of candidate services for every task, decreases the time to create composition strategies.

Cai et al. [18] proposed a cloud service composition technique depending upon multi granularity clustering, organizing services in the viewpoint of granularity for meeting user needs in service composition. The services disorder becomes multi granularity clustering that involves 3 stages: basic services clustering is depending upon multi granularity services clustering, correlation mining and message semantic similarity computing. The study illustrates that by using the presented technique, diverse requirements and user personalized are fulfilled and the performance of service composition is greatly improved. 


\section{The Proposed Model}

Fig. 1 showcases the overall working process of proposed F3L-WSCM model. The presented F3LWSCM model developed a novel composition WS technique for trip planning. The presented model combines with the GIS application of producing interactive interface for Tourism. The presented model involves three major modules namely discovery module, selection module, and execution module. Firstly, the discovery module enables the client to provide a query related to trip planning such as flight booking, hotels, car rentals, etc. The discovery module holds details related to flights, hotels, taxi services, etc. Besides, the presented F3L-WSCM model involves 4 input QoS parameters namely service cost, service availability, service response time, and user rating. Then, the FF algorithm gets executed to generate a set of WS composition plans which are then fed into the selection module. During the selection process, the FSC technique elects an optimal WS composition plan. Finally, at the execution plan, the chosen plan will be sent to the client and $\mathrm{CC}$ server for further processing.

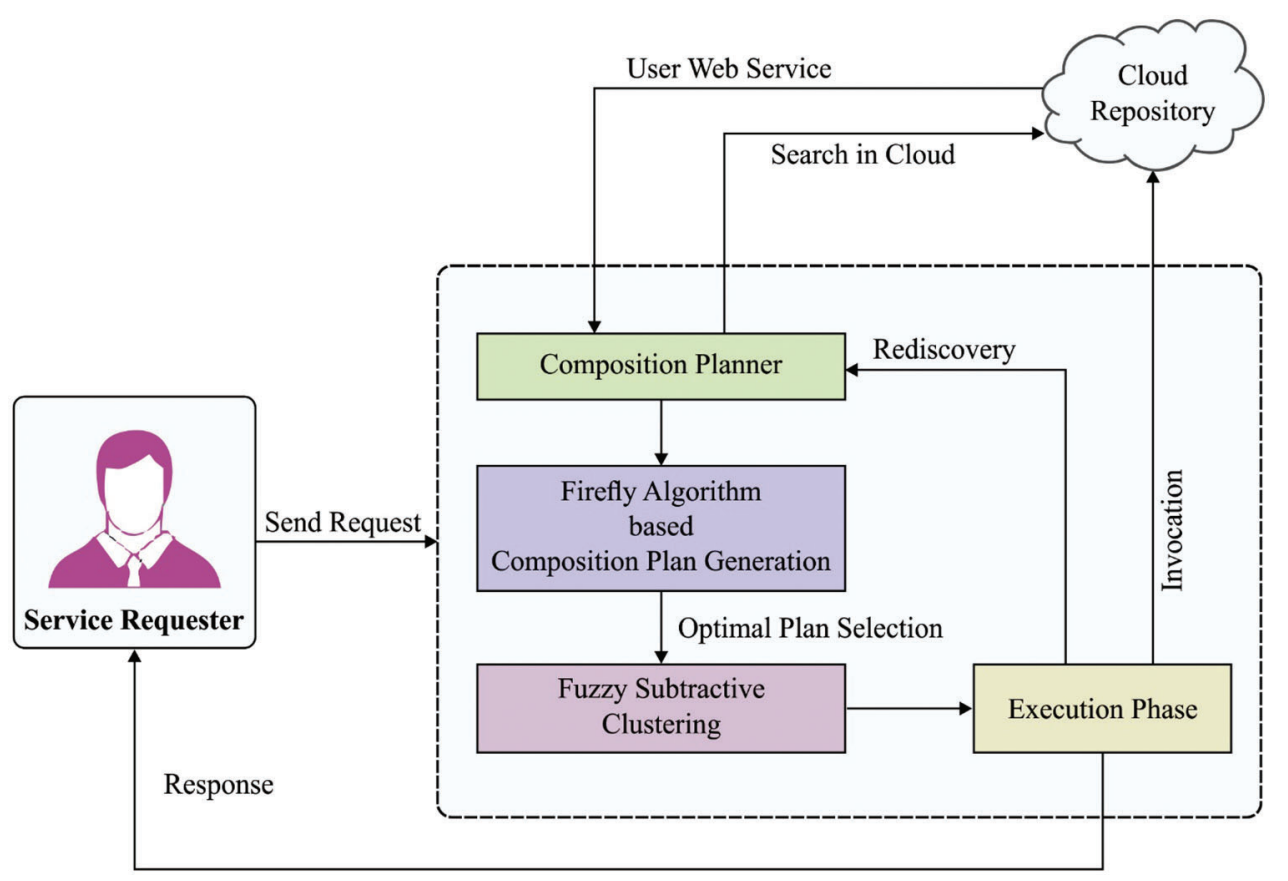

Figure 1: Working process of F3L-WSCM model

The aims of the WS selection from discovered services are depending upon their business requirements; they would go to the composition procedure. A set of detected WSs is separated into the division of services. This service is known as candidate service for a provided client request and their requirements. Henceforth, in created group, the division of services implements an essential process and other separations execute different types of tasks. Aforementioned, they realize a process as WS logic or service action. Therefore, in process selection, they must determine a group of candidate WSs $s_{i}, i \in[1 \ldots n]$ this could perform a set of processes $t_{j}, j \in[1 \ldots m]$.

The main objective is assuming a collection of candidates WS for every provided task, which is to decided that WS completes the provided task, this is the method of identifying services in the composition process. The QoS non-functional method which is composed of 4 variables for the quality of WSs method [19]: namely service availability, response time, user rating, and costs. All candidate services would attain a value for demonstrating this quality of WS standards. 


\section{i) Service Cost} j task

The cost quality $c_{i j}$ denotes the money that the service requester should pay for executing the service $\mathrm{i}$ by

$c_{i j}, \quad i \in[1 . . n], \quad j \in[1 . . m]$

Assume that $c_{i j}$ is undefined in case service i could not perform the t task.

ii) Service Availability

The availability quality $a_{i j}$ represents likelihood that the service is used and accessed. It denotes the number of service responses to a request and overall requests have created to the service. It is demonstrated as follows:

$a_{i j}=\frac{r e q_{i j}}{\operatorname{tot}_{i j}}$, tot $_{i j} \neq 0, i \in[1 \ldots n], j \in[1 \ldots m]$

iii) User rating

The user rating represents the measure of reliability. It is based on user experience by the service. Various end users could have distinct views regarding similar services. Reputation (or user rating) is determined by the average rank provided to the service by end user. The reputation of provided service is generally determined by:

$q_{\text {rep }}=\frac{\sum_{b=1}^{N} k_{b}}{N}$

iv) Service Response Time

The time quality $t_{i j}$ calculates the performance time among moment the request is sent and instant outcomes are attained $\mathrm{j}$ :

$R T=\sum t_{i j}, \quad i \in[1 \ldots n], \quad j \in[1 \ldots m]$

\subsection{Firefly Algorithm Based Composition Plan Model}

The FF Algorithm consists of nature inspired, optimization, metaheuristic method that is depending upon the social (i.e., flashing) behavior of fireflies/lighting bugs in the tropical temperature regions. According to the swarm behaviors like insects, fish, bird schooling [20]. Especially, the FF technique has several common features with other techniques that are depending upon swarm intelligence (SIs). In fact, it is easier in both implementation and concepts [21]. Based on current research, the technique is highly effective and outperforms different traditional methods like genetic algorithm (GA), to resolve various optimization challenges. The major benefit that it is utilizes mostly in real arbitrary numbers and depending upon global communication between the swarming particles (that is fireflies), and consequently, it appears highly efficient in multi-objective optimizations like WS composition plan generation. Fig. 2 illustrates the FF technique.

The FF technique consists of 3 specific guidelines that are depending upon main flashing features of actual fireflies. (1) Entire fireflies are unisex, and it proceeds near brighter and attractive ones nevertheless their sex. (2) The attractiveness degree of FF is equivalent to their brightness that reduces with the increase in distance from another FF because air absorbs light. When there are no brighter/attractive fireflies compared to specific one, it would move arbitrarily. (3) The brightness/light intensity of FF is 
defined by the objective function value of a provided problem. For WS composition plan generation problem, the light intensity is equivalent to the objective function value.

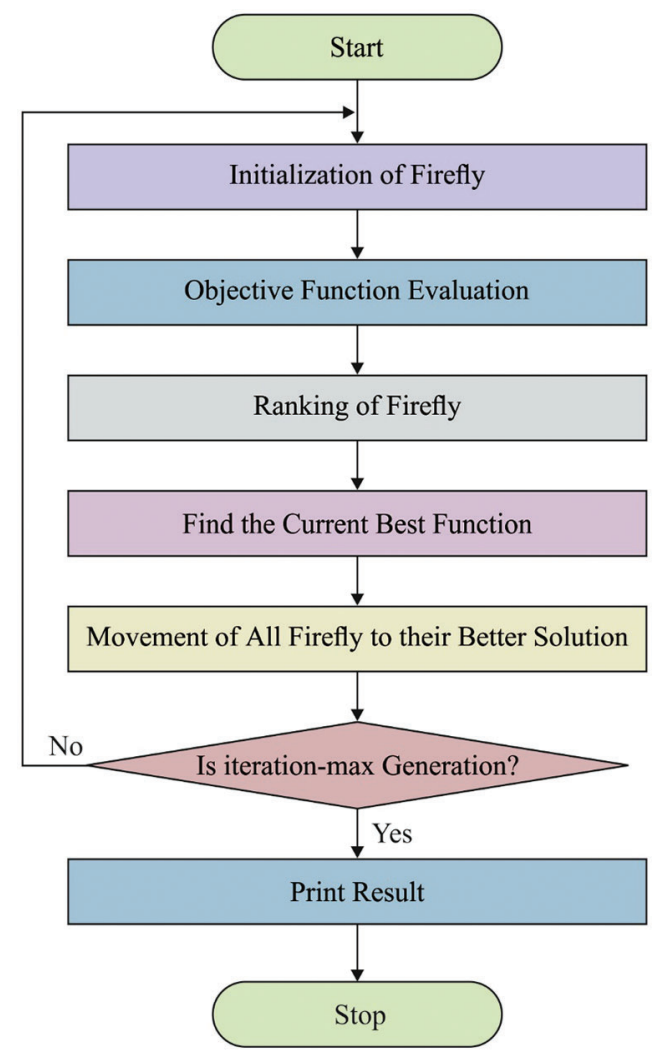

Figure 2: Flowchart of FF algorithm

Moreover, the intensity reduces with distance and it depends on inverse square law as provided in Eq. (5).

$I \prec \frac{1}{r^{2}}$

When the light is traversing in a medium with light absorption coefficient $\gamma$, later the light intensity at distance of $r$ in the source is provided using Eq. (6).

$I=I_{0} e^{-\gamma r^{2}}$

where $I_{0}$ refers the light brightness at the source [22]. Likewise, the intensity, $\beta$, is provided using Eq. (7).

$\beta=\beta_{0} e^{-\gamma r^{2}}$

The generalization intensity function for $\omega \geq 1$ is provided in Eq. (8). Actually, some monotonically reducing function is utilized as follows:

$\beta=\beta_{0} e^{-\gamma r^{\omega}}$

During this technique, an arbitrarily created possible solution known as FFs is allocated with light intensity depending upon its efficiency as the main function. This brightness is utilized for computing the 
intensity of FF that is directly proportional to their light intensity. Once the intensity/brightness of the solutions is allocated, all FFs follow FFs with optimal light intensity. For the intensity FF, it acts as the local search by arbitrarily from their neighborhood. Therefore, for $2 \mathrm{FFs}$, when the $F F_{j}$ is brighter than $F F_{i}$, then $F F_{i}$ is location towards $F F_{j}$ utilizing the upgrading equation provided in Eq. (9).

$x_{i}:=x_{i}+\underbrace{\beta_{0} e^{-\gamma r_{i j}^{2}}}_{=\beta}\left(x_{j}-x_{i}\right)+\alpha(\epsilon()-0.5)$

where $\beta_{0}$ implies the attraction of $x_{j}$ at $r=0$, in researchers suggested that $\beta_{0}=1$ for execution, $\gamma$ represents the algorithm parameter that defines the degree in that the upgrading model depends upon the distance among $2 \mathrm{FFs}, \alpha$ signifies the algorithm parameter for step length of arbitrary progress and $\epsilon(\quad)$ refers the arbitrary vector from uniform distribution with values among $[0,1]$. To intensity FF, $x_{b}$, the second expression in Eq. (9) is neglected, as provided in Eq. (10).

$x_{b}:=x_{b}+\mathrm{a}(\epsilon()-0.5)$

\section{Algorithm 1: Pseudo code of FF algorithm}

Establish algorithm parameters $(\alpha, \gamma)$

Establish model set-up (number of primary solutions and maximal iteration ( $N$, MaxGen))

Randomly create $N$ first solutions

for iteration = 1: MaxGen

Calculate the intensity, $I$

Sort the solution in order that, $I_{i} \geq I_{i-1}, \forall i$

$$
\begin{aligned}
& \text { for } i=1: n-1 \\
& \text { For } j=i+1: n \\
& \text { If } I_{j}>I_{i} \\
& \quad \text { move FF } i \text { towards FF } j
\end{aligned}
$$

end if

end for

end for

move FF $N,\left(x_{b}\right)$, arbitrarily

end for

Report the optimal solution,

These upgrades of the position of FFs maintain with iteration till an end condition is met. The end condition is maximal number of iterations, a tolerance in the better value when it is recognized or no enhancement is attained from the consecutive iterations.

\subsection{Fuzzy Subtractive Clustering Based Optimal Composition Plan Selection Model}

Once the set of WS composition plans are generated, the FSC technique gets executed to choose an optimal web service from the existing ones. Subtractive clustering (SC) considers better data points for defining the cluster center depends on the density of neighboring data points. Assume a group of $\mathrm{n}$ data points: $X=\left\{x_{1}, \quad x_{2}, \quad x_{\mathrm{n}}\right\}$, where, $x_{i}$ implies the vector from M-dimension space [23]. The SC technique contains the subsequent processes: 
Step 1: Initialize, $r_{a}, \eta$ with $\eta=\frac{r_{b}}{r_{a}}, \bar{\epsilon}$ and $\epsilon$.

Step 2: Compute the density to each data point utilizing Eq. (11):

$P_{i}=\sum_{j=1}^{n} e^{-\frac{4}{r} r_{a}^{2}\left\|x_{i}-x_{j}\right\|^{2}}$

where $P_{i}$ indicates the density of $i^{\text {th }}$ data point, $r_{\alpha}$ represents the positive constant determining a neighboring radius and $\|$.$\| refers the Euclidean distance. The data points with maximum density are elected as initial$ cluster center.

Step 3: The density of every data point is reviewed utilizing Eq. (12):

$P_{i}=P_{i}-P_{k}^{*} e^{-\frac{4}{r_{b}^{2}}\left\|x_{i}-x_{k}^{*}\right\|^{2}} ; i=1, \ldots, n$

where $r_{b}$ signifies the positive constant and $r_{b}=\eta^{*} r_{a}$ with an optimal choice of $\eta=1.5$.

Step 4: Assume $x^{*}$ is the data point with their density is maximum and equivalent $P^{*}$

- If $P^{*}>\bar{\epsilon} P^{r e f}: x^{*}$ is the new cluster center and jump to Step 3.

- Else if $P^{*}<\underline{\epsilon} P^{r e f}$ : back to Step 5 .

- Else:

- Consider $d_{\min }$ refers the minimum distances among $x^{*}$ and all earlier created cluster centers.

- If $\frac{d_{\min }}{r_{a}}+\frac{P^{*}}{P^{\text {ref }}} \geq 1: x^{*}$ is the new cluster center and jump to Step 3 .

- Else: $P\left(x^{*}\right)=0$ and elect $x^{*}$ with the next maximum density, $P\left(x^{*}\right)$, v go to step 4 .

Step 5: Display the clustering result.

If the membership degree of data point from all clusters can be defined using Eq. (13):

$\mu_{i k}=e^{-\frac{4}{r_{a}^{2}}\left\|x_{i}-x_{k}\right\|^{2}}$

During the SC technique, it requires a set of 4 parameters: acceptance ratio $\bar{\epsilon}$, reflection ratio $\epsilon$, cluster radius $r_{a}$ and squash factor $\eta$ (or $r_{b}$ ). The chosen parameters significantly affect to outcomes of clustering. When values of $\bar{\epsilon}$ and $\epsilon$ are huge, the count of cluster centers is diminished. So, these parameters are uncertainties in the $\mathrm{SC}$ technique.

Conversely, the SC evaluated potential of data point as cluster centers depend on the density of neighboring data point that really depends on the distance among the data points with residual data points. So, the SC contains different kinds of uncertainties as distance measure, parameters initialized as:

$P_{i}=\sum_{j=1}^{n} e^{-\frac{4}{r_{a}^{2}}\left(x_{j}-x_{i}\right)^{\frac{2}{m-1}}}$

When $x_{k}$ is the $k^{\text {th }}$ cluster location is potential $P_{k}^{*}$, later the potential of all data points is reviewed by subsequent equation:

$P_{i}=P_{i}-P_{k}^{*} e^{-\frac{4}{r_{b}^{2}}\left(x_{i}-x_{k}\right)^{\frac{2}{m-1}}} ; i=1, \cdots, n$

Afterward, the selection of value of the parameter $m$ is highly affected by outcomes of clustering. When $\mathrm{m}$ is lesser, the count of cluster centers is diminished. On the other hand, when $m$ is excessive, so many cluster centers are created. Also, with the alteration of fuzzifier parameters $m$, it can be simple for 
obtaining an optimal outcome of clustering which is not based on the parameter setting of SC. The fuzzified variable $m$ alters the outcome of the mountain function and therefore it considerably affects the clustering outcome. Using the fuzzified variable $m$, the dependency of the clustering outcomes can be reduced in the initial parameter values of the technique. With the adjusted variable $m$, an improved clustering outcome can be attained regardless of the parameter initialization. By the use of FSC technique, the optimal composition plan is selected from the availability of many composition plans for location identification.

\section{Performance Validation}

This section validates the experimental validation of the presented F3L-WSCM model on two benchmark datasets. The records in the dataset comprise user_ID, geographical location, and tips in English language. The records fitting to the identical object to form textual description of the object. Tab. 1 and Figs. 3 and 4 examines the efficiency analysis of the F3L-WSCM model with other existing methods under varying number of activities [15,24-26]. A detailed comparative result analysis is made with the Nearest Neighbor based Algorithm (NNB), Deviation Distance based Algorithm (DDB), and Collective Service Composition based Trip Search (CSCB).

Table 1: Analysis of existing with proposed model on dataset-1 in terms of efficiency

\begin{tabular}{lllll}
\hline Query time (ms)-dataset 1 & & & & \\
\hline No. of activities & F3L-WSCM & NNB & DDB & CSCB \\
\hline 3 & 4.05 & 5.55 & 7.04 & 16.78 \\
6 & 4.05 & 5.92 & 7.04 & 50.49 \\
9 & 4.80 & 5.92 & 7.42 & 75.58 \\
12 & 4.05 & 5.92 & 7.42 & 90.93 \\
15 & 4.80 & 6.29 & 8.17 & 135.12 \\
\hline Query time (ms)-dataset 2 & & & & \\
\hline No. of activities & F3L-WSCM & NNB & DDB & CSCB \\
\hline 3 & 4.45 & 6.18 & 7.22 & 13.10 \\
6 & 3.41 & 6.18 & 7.91 & 36.63 \\
9 & 3.06 & 6.18 & 9.64 & 64.31 \\
12 & 4.10 & 7.56 & 10.68 & 88.19 \\
15 & 4.10 & 7.56 & 11.37 & 127.29 \\
\hline
\end{tabular}

On the other hand, on examining the efficiency (query time) analysis of the proposed F3L-WSCM model on dataset-1, the F3L-WSCM model has accomplished improved outcomes by offering a lower query time under distinct activities. For instance, the F3L-WSCM model requires a minimal query time of 4.05 whereas the NNB, DDB, and CSCB models have needed a maximum query time of 5.55, 7.04, and 16.78 respectively. Followed by, the F3L-WSCM model requires a minimal query time of 4.05 whereas the NNB, DDB, and CSCB models have needed a maximum query time of 5.92, 7.04, and 50.49 respectively. Next to that, the F3L-WSCM model requires a minimal query time of 4.80 whereas 
the NNB, DDB, and CSCB models have needed a maximum query time of 5.92, 7.42, and 75.58 respectively. Simultaneously, the F3L-WSCM model requires a minimal query time of 4.05 whereas the NNB, DDB, and CSCB models have needed a maximum query time of 5.92, 7.42, and 90.93 respectively. Concurrently, the F3L-WSCM model requires a minimal query time of 4.80 whereas the NNB, DDB, and CSCB models have needed a maximum query time of $6.29,8.17$, and 135.12 respectively.

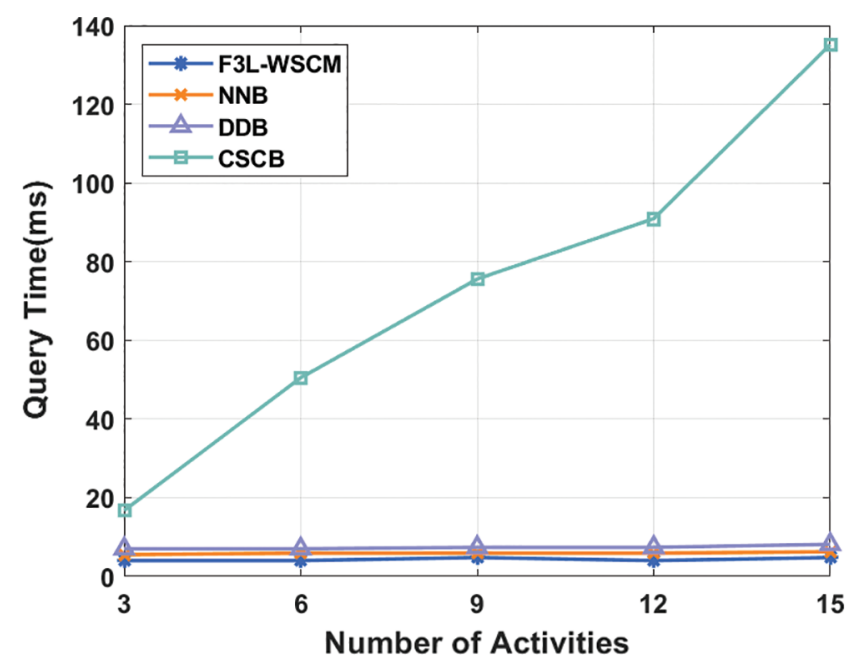

Figure 3: Query time analysis of F3L-WSCM model on dataset 1

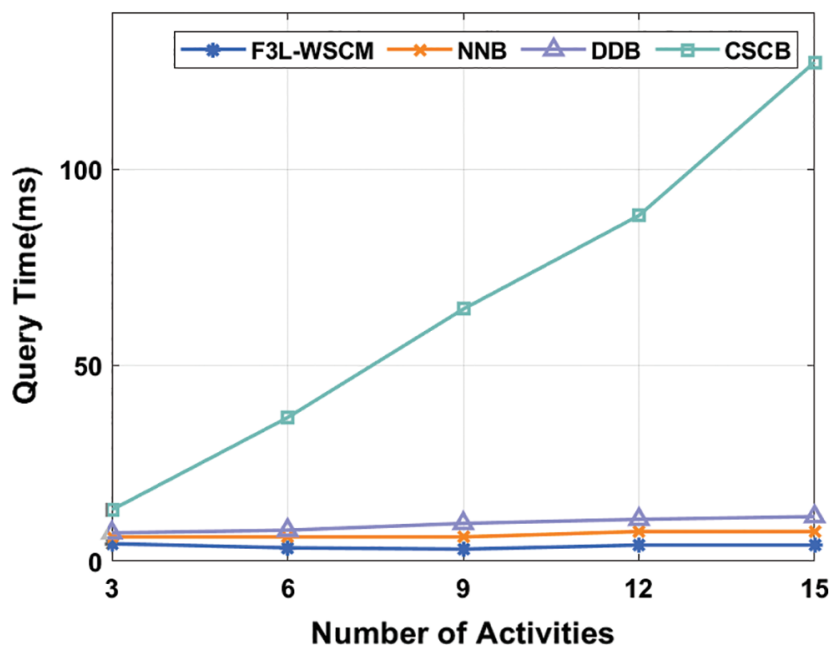

Figure 4: Query time analysis of F3L-WSCM model on dataset 2

Lastly, on investigating the efficiency (query time) analysis of the proposed F3L-WSCM model on the dataset-2, the F3L-WSCM model has accomplished improved outcome by offering a lower query time under distinct activities. For instance, the F3L-WSCM model requires a minimal query time of 4.45 whereas the NNB, DDB, and CSCB models have needed a maximum query time of $6.18,7.22$, and 13.10 respectively. Moreover, the F3L-WSCM model requires a minimal query time of 3.41 whereas the $\mathrm{NNB}$, DDB, and CSCB models have needed a maximum query time of $6.18,7.91$, and 
36.63 respectively. Furthermore, the F3L-WSCM model requires a minimal query time of 3.06 whereas the NNB, DDB, and CSCB models have needed a maximum query time of 6.18, 9.64, and 64.31 respectively. In line with this, the F3L-WSCM model requires a minimal query time of 4.10 whereas the NNB, DDB, and CSCB models have needed a maximum query time of 7.56, 10.68, and 88.19 respectively. Lastly, the F3LWSCM model requires a minimal query time of 4.10 whereas the NNB, DDB, and CSCB models have needed a maximum query time of $7.56,11.37$, and 127.29 respectively.

Tab. 2 and Figs. 5 and 6 inspects the efficiency analysis of the F3L-WSCM method with other existing methods under varying number of activities. On examining the efficiency analysis of the proposed F3LWSCM model on dataset-1, the F3L-WSCM model has accomplished improved outcomes by offering a higher distance to query under distinct activities. For instance, the F3L-WSCM model requires a maximum distance to query of 49.53 whereas the NNB, DDB, and CSCB models have needed a lower distance to query of $38.59,21.86$, and 6.66 respectively. Followed by, the F3L-WSCM model requires a maximum distance to query of 69.30 whereas the NNB, DDB, and CSCB models have needed a lower distance to query of $61.09,31.59$, and 11.53 respectively. Similarly, the F3L-WSCM model requires a maximum distance to query of 87.24 whereas the NNB, DDB, and CSCB models have needed a lower distance to query of $79.94,32.20$, and 14.57 respectively. Simultaneously, the F3L-WSCM model requires a maximum distance to query of 99.09 whereas the NNB, DDB, and CSCB models have needed a lower distance to query of $90.88,33.72$, and 20.65 respectively. Followed by, the F3L-WSCM model requires a maximum distance to query of 113.69 whereas the NNB, DDB, and CSCB models have needed a lower distance to query of $108.52,49.53$, and 21.56 respectively.

Table 2: Analysis of existing with proposed model on dataset-2 in terms of accuracy (Distance to query)

\begin{tabular}{lllll}
\hline \multicolumn{2}{l}{ Distance to query(km)-dataset 1} & & & \\
\hline No. of activities & F3L-WSCM & NNB & DDB & CSCB \\
\hline 3 & 49.53 & 38.59 & 21.86 & 6.66 \\
6 & 69.30 & 61.09 & 31.59 & 11.53 \\
9 & 87.24 & 79.94 & 32.20 & 14.57 \\
12 & 99.09 & 90.88 & 33.72 & 20.65 \\
15 & 113.69 & 108.52 & 49.53 & 21.56 \\
\hline Distance to query(km)-dataset 2 & & & \\
\hline No. of activities & F3L-WSCM & NNB & DDB & CSCB \\
\hline 3 & 28.29 & 22.69 & 19.54 & 5.89 \\
6 & 54.53 & 45.78 & 28.64 & 5.89 \\
9 & 93.72 & 85.32 & 34.94 & 6.94 \\
12 & 107.37 & 97.92 & 50.68 & 6.59 \\
15 & 129.76 & 124.86 & 75.53 & 6.59 \\
\hline
\end{tabular}

Besides, on analyzing the accuracy (distance to query) analysis of the proposed F3L-WSCM method on the dataset-2, the F3L-WSCM model has accomplished improved results by offering a higher distance to query under distinct activities. For instance, the F3L-WSCM model requires a higher distance to query of 28.29 whereas the NNB, DDB, and CSCB models have needed a reduced distance to query of 22.69, 
19.54, and 5.89 correspondingly. In line with, the F3L-WSCM method requires a maximal distance to query of 54.53 whereas the NNB, DDB, and CSCB models have needed a lower distance to query of 45.78, 28.64, and 5.89 correspondingly. In addition, the F3L-WSCM model requires a maximum distance to query of 93.72 whereas the NNB, DDB, and CSCB models have needed a lower distance to query of 85.32 , 34.94 , and 6.94 respectively.

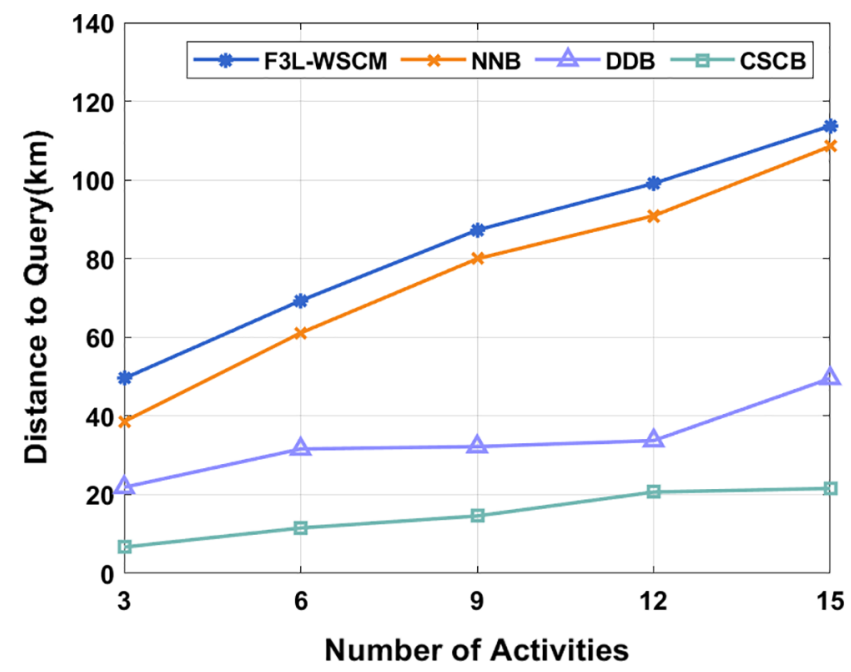

Figure 5: Distance to query time analysis of F3L-WSCM model on dataset 1

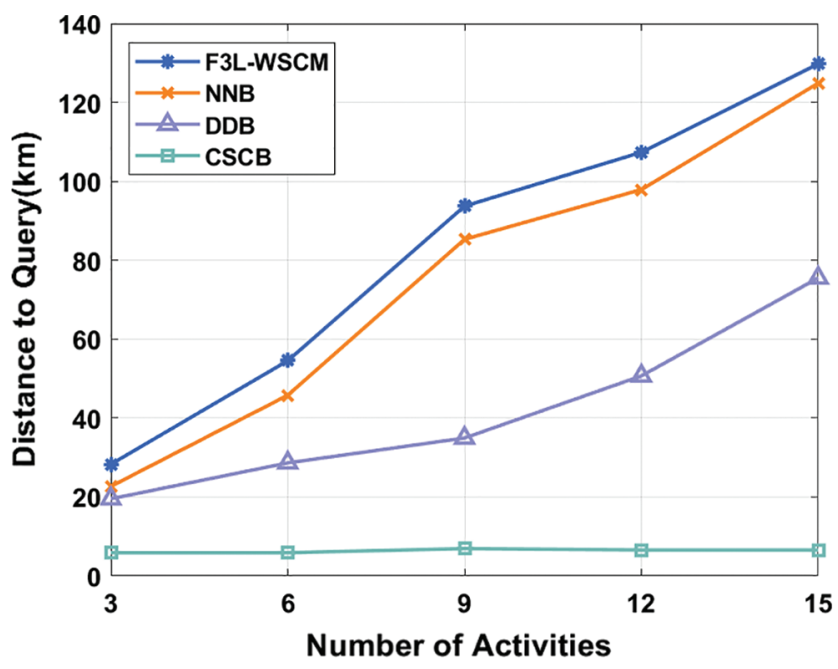

Figure 6: Distance to query time analysis of F3L-WSCM model on dataset 2

Concurrently, the F3L-WSCM technique requires a higher distance to query of 107.37 whereas the $\mathrm{NNB}$, DDB, and CSCB models have needed a lower distance to query of 97.92, 50.68, and 6.59 respectively. Also, the $\mathrm{F} 3 \mathrm{~L}-\mathrm{WSCM}$ methodology requires a superior distance to query of 129.76 whereas the NNB, DDB, and CSCB models have needed a minimum distance to query of 124.86 , 75.53 , and 6.59 correspondingly. From the experimental results, it is guaranteed that the proposed F3LWSCM model is found to be effective over the existing methods in a significant way. 


\section{Conclusion}

This study has introduced a novel F3L-WSCM in a CC environment for effective location awareness. The presented F3L-WSCM model primarily allows the client to provide a query related to location awareness. Next, the F3L-WSCM technique executes the FF algorithm that is inspired by the flashing patterns of fireflies for WS composition plan generation. Furthermore, the presented F3L-WSCM model involves four input QoS parameters namely service cost, service availability, service response time, and user rating. Next, the FSC technique is applied to pick the best composition plan from the available composite plans. A series of simulations were performed on benchmark dataset to demonstrate the promising results of the F3L-WSCM model over the existing methods. The obtained experimental results highlighted the improved performance of the F3L-WSCM model over the other methods in terms of different measures. In future, the presented F3L-WSCM model can be realized in real time applications.

Funding Statement: The authors received no specific funding for this study.

Conflicts of Interest: The authors declare that they have no conflicts of interest to report regarding the present study.

\section{References}

[1] M. Yaghoubi and A. Maroosi, "Simulation and modeling of an improved multi-verse optimization algorithm for QoS-aware web service composition with service level agreements in the cloud environments," Simulation Modelling Practice and Theory, vol. 103, pp. 102090, 2020.

[2] C. Ramalingam and P. Mohan, "Addressing semantics standards for cloud portability and interoperability in multi cloud environment," Symmetry, vol. 13, no. 2, pp. 317, 2021.

[3] C. Li, J. Li, H. Chen and A. A. Heidari, "Memetic harris hawks optimization: Developments and perspectives on project scheduling and qos-aware web service composition," Expert Systems with Applications, vol. 171, pp. 114529, 2021.

[4] F. Dahan, K. E. Hindi, A. Ghoneim and H. Alsalman, "An enhanced ant colony optimization based algorithm to solve qos-aware web service composition," IEEE Access, vol. 9, pp. 34098-34111, 2021.

[5] F. Seghir and G. Khababa, "Fuzzy teaching learning based optimization approach for solving the QoS-aware web service selection problem in uncertain environments," Journal of Ambient Intelligence and Humanized Computing, pp. 1-31, 2021.

[6] D. Paulraj and M. Dineshkumar, "An efficient key policy attribute based encryption scheme in cloud computing," International Journal for Scientific Research \& Development, vol. 9, no. 2, pp. 1-14, 2017.

[7] S. -Y. Hwang, H. Wang, J. Tang and J. Srivastava, "A probabilistic approach to modeling and estimating the QoS of web-services-based workflows," Information Sciences, vol. 177, no. 23, pp. 5484-5503, 2007.

[8] M. Buvanesvari, J. Uthayakumar and J. Amudhavel, "Fuzzy based clustering to maximize network lifetime in wireless mobile sensor networks," Journal of Advanced Research in Dynamical and Control Systems, vol. 12, pp. 2156-2167, 2017.

[9] M. Zhu, G. Fan, J. Li and H. Kuang, "An approach for QoS-aware service composition with graphplan and fuzzy logic," Procedia Computer Science, vol. 141, pp. 56-63, 2018.

[10] H. Alhadithy and B. Al-Shargabi, "Fuzzy rule based web service composition in cloud," in Proc. of the First Int. Conf. on Data Science, E-Learning and Information Systems, Madrid Spain, pp. 1-4, 2018.

[11] F. Rhimi, S. Ben Yahia and S. Ben Ahmed, "Balancing between local and global optimization of web services composition by a fuzzy transactional-aware approach," in Proc. of the 11th Int. Joint Conf. on Software Technologies, Lisbon, Portugal, pp. 75-82, 2016.

[12] Z. Ma and K. Ben, "A semantic service composition method based on fuzzy colored petri net," in Advances in Computer Science and Education Applications. Proceedings: Communications in Computer and Information Science Book Series (CCIS, Volume 202), Springer, Berlin, Heidelberg, pp. 396-404, 2011. 
[13] A. K. Sangaiah, G. -B. Bian, S. M. Bozorgi, M. Y. Suraki and A. A. R. Hosseinabadi, "A novel quality-of-serviceaware web services composition using biogeography-based optimization algorithm," Soft Computing, vol. 24, no. 11, pp. 8125-8137, 2020.

[14] A. S. d. Silva, E. Moshi, H. Ma and S. Hartmann, "A QoS-aware web service composition approach based on genetic programming and graph databases," in Int. Conf. on Database and Expert Systems Applications, DEXA 2017: Database and Expert Systems Applications. Proc.: Lecture Notes in Computer Science Book Series (LNCS, Volume 10439), Springer, Cham, pp. 37-44, 2017.

[15] J. Xu, J. Chen, R. Zhou, J. Fang and C. Liu, "On workflow aware location-based service composition for personal trip planning," Future Generation Computer Systems, vol. 98, pp. 274-285, 2019.

[16] H. Wang, M. Gu, Q. Yu, Y. Tao, J. Li et al., "Adaptive and large-scale service composition based on deep reinforcement learning," Knowledge-Based Systems, vol. 180, pp. 75-90, 2019.

[17] D. Mallayya, B. Ramachandran and S. Viswanathan, "An automatic web service composition framework using QoS-based web service ranking algorithm," The Scientific World Journal, vol. 2015, pp. 1-14, 2015.

[18] H. Cai and L. Cui, "Cloud service composition based on multi-granularity clustering," Journal of Algorithms \& Computational Technology, vol. 8, no. 2, pp. 143-161, 2014.

[19] R. Sethuraman, T. Sasiprabha and A. Sandhya, "An effective QoS based web service composition algorithm for integration of travel \& tourism resources," Procedia Computer Science, vol. 48, pp. 541-547, 2015.

[20] M. Prakash and C. Saravana Kumar, "An authentication technique for accessing de-duplicated data from private cloud using one-time password," International Journal of Information Security and Privacy, vol. 11, no. 2, pp. 110, 2017.

[21] T. Apostolopoulos and A. Vlachos, "Application of the firefly algorithm for solving the economic emissions load dispatch problem," International Journal of Combinatorics, vol. 2011, pp. 1-23, 2011.

[22] W. A. Khan, N. N. Hamadneh, S. L. Tilahun and J. M. Ngnotchouye, "A review and comparative study of firefly algorithm and its modified versions," Optimization Algorithms-Methods and Applications, pp. 281-313, 2016.

[23] L. T. Ngo and B. H. Pham, "A type-2 fuzzy subtractive clustering algorithm," in Mechanical Engineering and Technology. Proceedings: Advances in Intelligent and Soft Computing Book Series (AINSC, Volume 125), Springer, Berlin, Heidelberg, pp. 395-402, 2012.

[24] R. Punithavathi, C. Kurangi, S. P. Balamurugan, I. V. Pustokhina, D. A. Pustokhin et al., "Hybrid bwo-iaco algorithm for cluster based routing in wireless sensor networks," Computers, Materials \& Continua, vol. 69, no. 1, pp. 433-449, 2021.

[25] M. S. Maharajan, T. Abirami, I. V. Pustokhina, D. A. Pustokhin and K. Shankar, "Hybrid swarm intelligence based QoS aware clustering with routing protocol for wsn," Computers, Materials \& Continua, vol. 68, no. 3, pp. 2995-3013, 2021.

[26] G. Kadiravan, P. Sujatha, T. Asvany, R. Punithavathi, M. Elhoseny et al., "Metaheuristic clustering protocol for healthcare data collection in mobile wireless multimedia sensor networks," Computers, Materials \& Continua, vol. 66, no. 3, pp. 3215-3231, 2021. 